Es wird befürchtet, dass die E-Card dazu führen kann, dass die Arzt-PatientenBeziehung durch die fortschreitende Technisierung und Bürokratisierung ins Hintertreffen gerät. Ein Blick auf die Karte reicht, um an wichtige Informationen zu kommen, ohne den Patienten zu sprechen oder überhaupt gesehen zu haben.

Vor diesem Hintergrund ist es nicht verwunderlich, dass immer mehr Verbände die Ärzteschaft dazu aufrufen, die Einführungstests der Gesundheitskarte zu boykottieren. Das vorliegende Konzept würde durch die Ärzteschaft und die
Versichertenbeiträge subventioniert und die damit befasste Industrie sahne ab, so der Vorwurf. Die Datensicherheit und der Umgang mit sensiblen Patientendaten kämen, wenn überhaupt, erst an zweiter Stelle.

Aber wie der EBM 2000 plus und die Gesundheitsreform so wird auch die endgültige Einführung der E-Card von der Politik abgenickt werden. Die Vertragsärzte werden als perfektionierte Krötenschlucker einen Großteil der Zeche übernehmen müssen.

Dr. Werner Kersten, Moers

\title{
Körperliche Aktivität und Heuschnupfenprävalenz
}

\author{
Ein Charakteristikum moderner Lebensweise ist die Reduktion \\ körperlicher Belastung zugunsten sitzender Beschäftigung. Könnte \\ diese Tatsache auch zur Zunahme allergischer Erkrankungen in den \\ letzten Jahrzehnten beigetragen haben?
}

$\mathrm{F}$ orscher vom GSF - Forschungszentrum für Umwelt und Gesundheit in Neuherberg bei München wollten einem möglichen Zusammenhang zwischen körperlicher Inaktivität und der Prävalenz von Heuschnupfen auf die Spur kommen. Sie begannen ihre Untersuchung in den Jahren 1992/93 mit 2.470 Kindern unterschiedlichen Alters und verfolgten deren gesundheitliche Entwicklung in drei- bis vierjährigen Abständen weiter fort. Dafür griffen sie auf klinische sowie auf Laboruntersuchungen zurück und setzten speziell entwickelte Fragebögen ein. Die Studie wurde 2005 abgeschlossen. Über die körperliche Betätigung der Kinder holten die Autoren sich Auskunft bei den Eltern, wobei zwischen völliger Inaktivität und teilweiser sowie regelmäßiger Sportausübung unterschieden wurde.

Im Ergebnis zeigte sich, dass schon bei der Eingangsuntersuchung, ebenso wie bei jeder der folgenden Kontrolluntersuchungen, die Prävalenz der allergischen Rhinitis bei den körperlich Inaktiven deutlich höher lag. Selbst bei nur moderater sportlicher Betätigung hatten die körperlich Aktiven eindeutig gesundheitliche
Vorteile. Wie solche Effekte zustande kommen, bleibt vorerst spekulativ. Möglicherweise spielt die für körperliche Belastung nachgewiesene Reduktion von Entzündungsmediatoren eine Rolle.

\section{Fazit}

Körperliche Aktivität senkt bei Kindern signifikant das Risiko, an Heuschnupfen zu erkranken. Somit gilt auch aus allergologischer Sicht, dass Kinder schon in frühen Jahren zu regelmäßiger sportlicher Betätigung motiviert werden sollten. Für Allergiker können sich zwar Einschränkungen - beispielsweise durch ein bestehendes Asthma bronchiale - ergeben, keinesfalls sollte dies aber zur Aufgabe jeglicher sportlicher Betätigung führen. In solchen Fällen wird die Betreuung durch einen kompetenten und sportmedizinisch erfahrenen Allergologen von entscheidender Bedeutung sein.

Dr. Dieter Bruchhausen, Wuppertal

\section{Literatur}

Kohlhammer Y, Zutavern A, Rzehak P, Woelke G, Heinrich J. Influence of physical inactivity on the prevalence of hay fever. Allergy 2006; 61: $1310-5$ 